\title{
Understanding the effect of confinement in scanning spreading resistance microscopy measurements
}

\author{
Komal Pandey ${ }^{1,2,}$, Kristof Paredis ${ }^{1}$, Alexander J. Robson ${ }^{3,4}$, and Wilfried Vandervorst ${ }^{1,2}$ \\ ${ }^{1}$ Imec, Kapeldreef 75, 3001, Leuven, Belgium \\ ${ }^{2}$ Quantum Solid-State Physics, KU Leuven, Celestijnenlaan 200D, B-3001 Leuven, Belgium \\ ${ }^{3}$ Department of Physics, Lancaster University, Lancaster, LA1 4YB, United Kingdom \\ ${ }^{4}$ Lancaster Material Analysis, Lancaster University, Lancaster, LA1 4YB, United Kingdom \\ *Corresponding Author email: komal.pandey@imec.be
}

\begin{abstract}
Scanning spreading resistance microscopy (SSRM) is a powerful technique for quantitative two-and three-dimensional carrier profiling of semiconductor devices with sub-nm spatial resolution. However, considering the sub-10nm dimensions of advanced devices and the introduction of three-dimensional architectures like FinFETs and nanowires, the measured spreading resistance is easily impacted by parasitic series resistances present in the system. The limited amount of material, presence of multiple interfaces and confined current paths may increase the total resistance measured by SSRM beyond the expected spreading resistance, which can ultimately lead to an inaccurate carrier quantification. Here, we report a TCAD assisted experimental study to identify the different parameters affecting the SSRM measurements in confined volumes. Experimentally, the two-dimensional current confinement is obtained by progressively thinning down uniformly doped blanket SOI wafers using scalpel SSRM. The concomitant SSRM provides detailed electrical information as a function of depth up to oxide interface. We show that the resistance is most affected by the interface traps in case of a heterogeneous sample, followed by the intrinsic resistance of the current carrying paths. Further, we show that accurate carrier quantification is ensured for typical back-contact distances of $1 \mu \mathrm{m}$ if the region of interest is at least 9 times larger than the probe radius.
\end{abstract}

\section{Introduction}

The continuous miniaturization of device dimensions, their transition from planar to threedimensional (3D) architectures, and the introduction of novel materials in the gate stack, channel and source/drain regions have placed very stringent requirements on their metrology. For instance, an appropriate technique is needed to probe the active dopant density within a 3D device (such as FinFET, tunnel FET or nanowire) such that the underlying physics of dopant incorporation, diffusion and activation can be understood and serve as a feedback in 
the development of the doping process ${ }^{1}$, which is in the end very crucial in achieving high device performance. This implies that probing carriers in today's sub-10 nm node requires a technique, which can provide a quantitative 3D distribution with sub-nm spatial resolution with a large dynamic range (spanning more than 5 orders of magnitude). Scanning Spreading Resistance Microscopy (SSRM), though inherently a 2D technique, fulfils all the aforementioned requirements ${ }^{2-7}$ and was recently expanded towards 3D applications ${ }^{8-10}$. In essence, it relies on measuring the resistance between the tip of a scanning probe and a large current collecting contact, created somewhere on the device of interest. The measured resistance is dominated by the current spreading in the nanoscale probe contact, therefore termed spreading resistance $\left(R_{\text {spr }}\right)$, and can be proven to have a direct relation with the local sample resistivity $\left(\rho_{\text {sample }}\right)$. The current confinement in this probe contact, which provides a high spatial resolution, and the direct quantification in terms of $\rho_{\text {sample }}$ are the key factors to the success of the SSRM technique ${ }^{11-14}$. To achieve this, one needs to ensure that the spreading resistance dominates the total measured resistance ( $R_{\text {Total }}$ ), which has been achieved in the past by minimizing all the parasitic resistances present in a typical SSRM set-up ${ }^{13}$. For planar device applications, the series resistance of the conduction path towards the current collecting contact is negligible relative to the spreading resistance due to the relaxed dimensions. However, as the device dimensions are getting smaller, this assumption is no longer valid in many cases. The interpretation of the measurement on confined volumes, therefore, becomes challenging due to the increased parasitic resistances originating from the scaled geometry of the device and the narrow conduction paths towards the current collection contact. For example, the intrinsic series resistance of a long and narrow FinFET structure becomes large enough that it cannot be ignored anymore. Experimentally, creating a reliable current collecting contact on these confined structures becomes a complex task as well. In many cases, as a result of the complex integration schemes employed in today's device fabrication process, the contact is placed at locations whereby the current needs to travel through multiple interfaces and junctions whose effect on $R_{\text {Total }}$ cannot be ignored anymore. Moreover, the formation of an ideal ohmic contact on reduced contact areas and on new materials becomes less obvious. All these effects increase the parasitic resistance and hinder the quantification. Indeed, the quantification of SSRM data is based on the use of a calibration curve, obtained on a large uniformly doped sample where these parasitic resistances are absent. Therefore, the conversion based on such a calibration curve becomes inaccurate. Moreover, the relation between $R_{\text {spr }}$ and $\rho_{\text {sample }}$ (see eq. 1 where $a$ is the electrical contact radius) has been derived for the case where the current spreading is not restricted by the dimensions of the sample probed $^{15}$. When the device size becomes comparable to the probe-sample nano contact, the direct relation between $R_{s p r}$ and $\rho_{\text {sample }}$ needs to be re-evaluated. 


$$
R_{\text {spr }}=\frac{\rho_{\text {sample }}}{4 a}
$$

As a first step towards understanding the deviation from eq.1, we conducted a fundamental study to identify the main contributors affecting the SSRM measurements when the sample dimensions are predominantly $2 \mathrm{D}$, a situation typically occurring when measuring FinFET or GAA devices ${ }^{16-18}$. A good starting point is a thin Silicon on Insulator (SOI) sample where the current flow and its spreading is confined within the thickness of the top thin silicon layer. To establish the dependence on the Si thickness, we started from a fairly thick sample and gradually reduced the sample thickness by performing Scalpel SSRM measurements ${ }^{8}$ and thus recorded the resistance as function of the Si thickness. Further, we used finite element simulations to understand the experimental results.

\section{Experimental Details and Simulation Approach}

The samples studied in this work are commercially available blanket SOI wafers from SOITEC with top Si and underlying oxide thicknesses of $220.0 \mathrm{~nm}$ and $2.0 \mu \mathrm{m}$, respectively. The top Si layer is (100) oriented and has a uniform Boron doping of $5 \times 10^{15} \mathrm{~cm}^{-3}$ (resistivity from 8.5-11.5 $\Omega-\mathrm{cm})$. For the highly doped $\mathrm{Si}$, the same SOI wafer was dual implanted with Phosphorus. The first and the second implantation steps were performed at a dose of $3.0 \times 10^{15} \mathrm{~cm}^{-3}$ with implant energies of 80.0 and $140.0 \mathrm{keV}$, respectively. After performing rapid thermal annealing at $1075^{\circ} \mathrm{C}$ a uniform active Phosphorus doping of $5.0 \times 10^{19} \mathrm{~cm}^{-3}$ was obtained. A Bruker Dimension ICON Atomic Force Microscope (AFM) equipped with SSRM module was used to carry out all the SSRM (or scalpel SSRM) measurements reported in this paper. The instrument was enclosed in a glovebox filled with non-reactive argon gas to provide a low humidity environment. We used imec boron doped Full Diamond Tips (FDT) ${ }^{19}$ with an average spring constant of $27 \mathrm{~N} / \mathrm{m}$ for all measurements. These tips are extremely hard and wear resistant and enable us to perform reliable SSRM measurements during the entire scalpel run. Both the sample structure and scalpel SSRM process are schematically depicted in figure 1a and $1 \mathrm{~b}$. Note that a current collection contact was placed far outside the thinned region by scratching with a diamond pen and then depositing conductive silver paste inside the groove. This ensures that the SSRM resistance can only be influenced by the series resistance through the thinned region and the changes in the current spreading but not by a change in back contact resistance. The material removed during the scalpel process gets accumulated at the sides of the scanned area and a crater of same size is formed. Celano et al. ${ }^{20}$ have 
reported that this debris gets redeposited into the crater and reduces the removal rate. To overcome this issue, we started with a relatively large scan area $\left(7 \times 7 \mu \mathrm{m}^{2}\right)$, which was progressively decreased (up to $1 \times 1 \mu \mathrm{m}^{2}$ ) when a decrease in the removal rate is noticed. This results in a staircase like topography as shown in figure $2 \mathrm{~b}$. In order to avoid any discrepancy arising from an uneven removal of the material, we used only the data corresponding to a central area of $500 \times 500 \mathrm{~nm}^{2}$ in all the further analysis. To ensure an accurate calculation of the remaining top Si layer thickness, AFM topography measurements were carried out after every 2-3 SSRM scans. The removed depth as measured from these topographical images is then divided by the number of scans to extract an average removal rate per scan. In this manner we obtain the removed depth corresponding to each 2D SSRM image, which after subtracting from the initial thickness of the top Si layer $(220 \mathrm{~nm})$ represents the remaining thickness of the top Si layer. If not stated otherwise, all the scalpel runs were performed at a sample bias of $0.5 \mathrm{~V}$ (and $-0.5 \mathrm{~V}$ ) for $\mathrm{p}$-type (and $\mathrm{n}$-type) and the tip was always grounded. All the parameters that can affect the measured resistance, i.e. the force applied on the cantilever, the voltage applied on the sample with respect to the grounded probe and the intrinsic probe resistance, were kept constant during the entire scalpel run. Of course, the tip degradation during the scalpel process may also impact the results. This impact is avoided by measuring on another location with the same probe and parameters, immediately after the scalpel process. Only if the same resistance is recorded as in the very first scan within the experimental error (25\%), the measurement run is included in the analysis, all others are discarded. During the scalpel process the surface roughness underwent a variation from 0.12 $\mathrm{nm}$ (pristine surface) to $0.29 \mathrm{~nm}$ (after first scans) to $0.07 \mathrm{~nm}$ (after the final scan) as characterized by tapping mode AFM. This trend reflects the expected dependency on the scan line density ${ }^{20}$, i.e. roughness increases with scanning at low scan line density and decreases at high density. There is no direct between the roughness trend and the observed resistance.

For the simulations, the Synopsys's Sentaurus structure editor ${ }^{21}$ was used to build a cylindrical geometry, as depicted in figure 1c, similar to the SOI sample used in the experiments. The radius of the cylinder was taken as $1 \mu \mathrm{m}$ to avoid any confinement in the lateral dimensions. Sentaurus device simulations ${ }^{22}$ corresponding to different cylinder heights $(H)$ were performed to mimic the scalpel SSRM process. Poisson's equation along with continuity equations for both electrons and holes are solved to obtain the various parameters like total current, electron \& hole density maps etc. The bias is progressively increased up to $0.5 \mathrm{~V}$ (same as used in scalpel SSRM) while quasi stationary equations are solved in each step. Note that the probe contact is placed at the centre of the top surface of the cylinder while the back contact is at the cylinder's curved surface (shown in outer grey color). As the purpose of these simulations is to understand the effect of different resistive components contributing into the SSRM 
measurements, further details on the simulation such as the properties of different contacts and interfaces will be explained later in this text as and when necessary.

a)
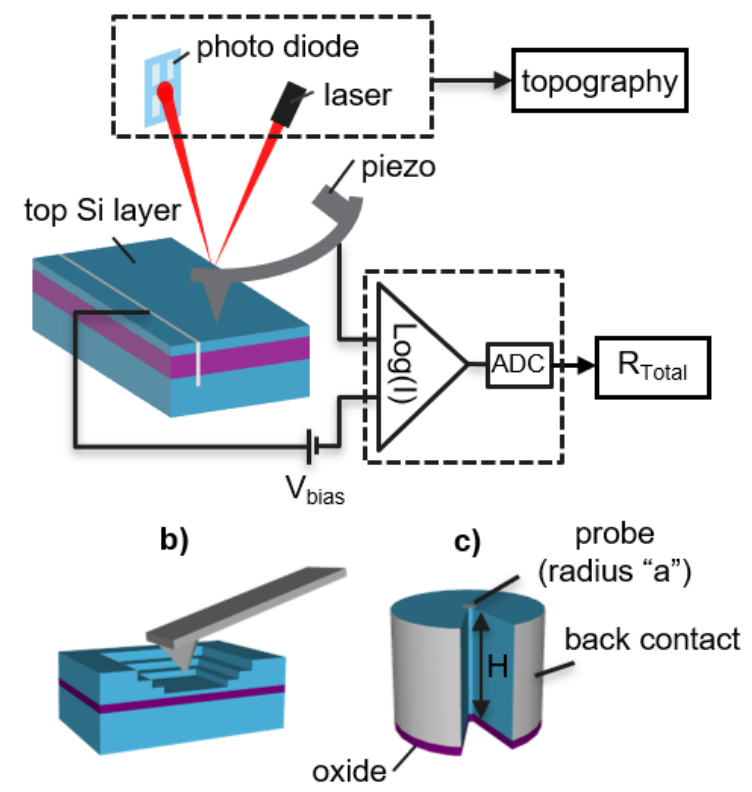

Figure 1: a) Schematic of the scalpel SSRM set-up. b) Schematic of the scalpel-SSRM method where the probe is continuously scanned on the sample at very high force leading to a crater formation. A staircase like geometry is obtained by progressively decreasing the scan size. c) Schematic of the simulated cylindrical geometry.

\section{Results and Discussion}

A systematic study of the effects of geometrical confinement on SSRM measurements is conducted using scalpel SSRM where we thin down the top Si layer of the selected SOI sample by scanning an area at high force and simultaneously sensing the SSRM response on the resulting layer. The scalpel process is stopped when all the material from the top Si layer is etched away and the probe touches the highly resistive oxide layer underneath. As the current is confined within the top Si layer throughout the scalpel process, this experiment provides a measure of the effect of the Si thickness on the SSRM measurements. The data presented in figure 2 represents a complete scalpel SSRM run. The top Si layer in the presented sample was doped with a boron concentration of $5 \times 10^{15} \mathrm{~cm}^{-3}$. The $2 \mathrm{D}$ resistance maps obtained at successive depths are shown in figure 2a. It is evident here that with the increasing number of scans the total resistance measured by SSRM remains almost constant until the $9^{\text {th }}$ scan after which it increases continuously. After converting the scan number to the remaining thickness (of top Si layer), we obtain the evolution, shown in figure $2 b$, of the 
measured SSRM resistance as a function of top Si thickness. Each data point in this graph represents the average resistance measured at the central area of dimensions $500 \times 500 \mathrm{~nm}^{2}$, as indicated by dotted squares in figure $2 a$. We notice here that as the film thickness decreases from $220 \mathrm{~nm}$ to $80 \mathrm{~nm}$, the measured resistance remains constant. With further reduction in the film thickness the measured resistance shows an increase of many orders of magnitude. The resulting 2D and 3D topography after a scalpel run is also shown in figure 2c$2 e$. Note that in figure $2 b$ we report only one scalpel run while multiple scalpel runs, which are shown in figure $8 \mathrm{a}$, were performed on the same sample.
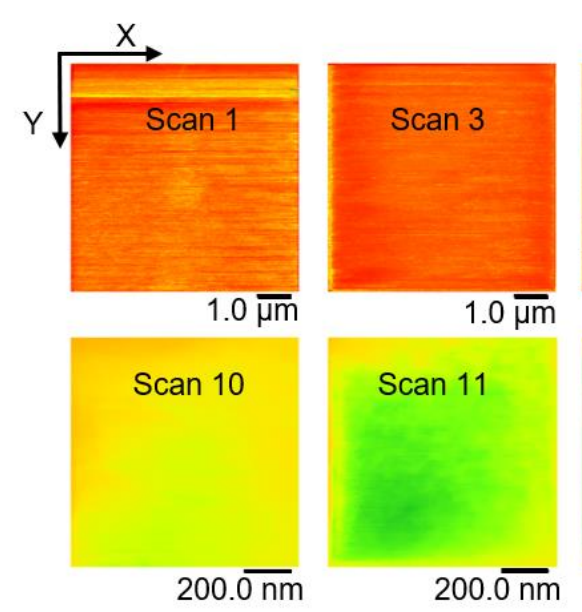

a)

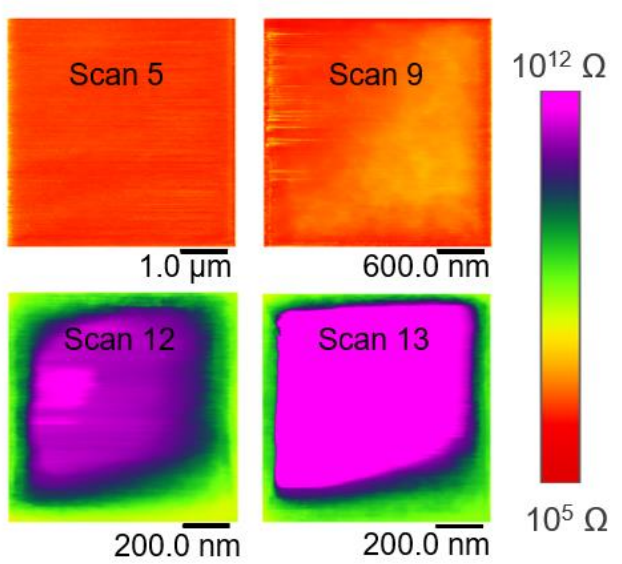

b)
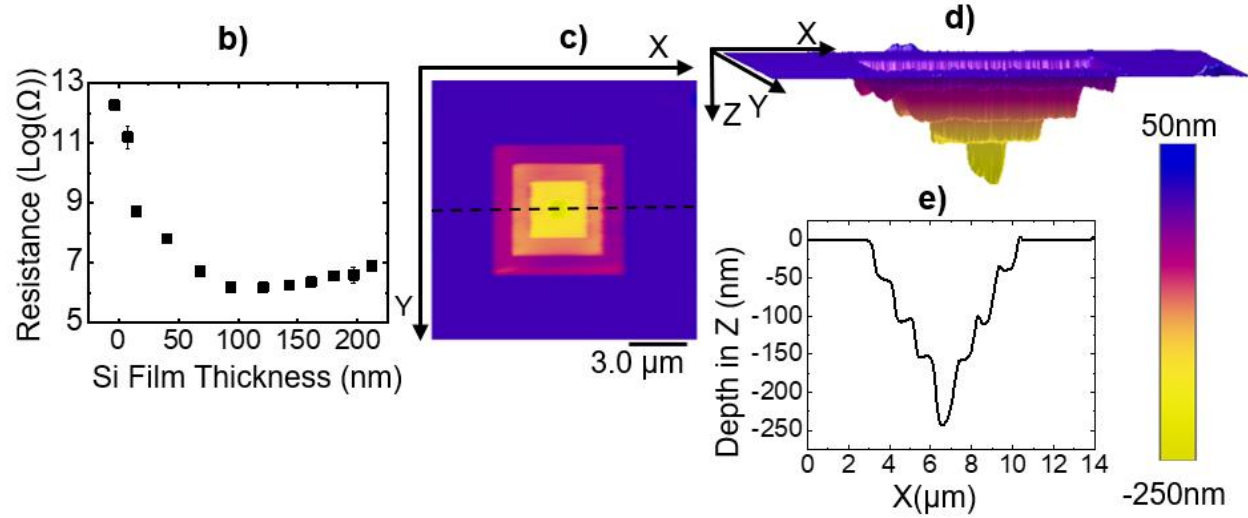

Figure 2: a) Multiple 2D scans obtained at different depths (or top Si film thicknesses) during scalpel process. The chosen dotted area at the centre is used to analyse the data. b) The average $R_{\text {Total }}$ is plotted as a function of top Si film thickness. c) A 2D and d) 3D topography image taken at the end of the scalpel run is also shown. e) Line scan taken across the dashed line (in black) shows a staircase like geometry produced due to progressively reducing the scan size during the scalpel process. 
Since SSRM measures the total resistance $\left(R_{\text {Total }}\right)$ between the probe and the back contact, we can now write the different resistive components present in the current path during our experiment as:

$$
R_{\text {Total }}=R_{\text {spr }}+R_{\text {sample }}+R_{\text {probe }}+R_{\text {probe-contact }}+R_{\text {back-contact }}
$$

where, $R_{s p r}$ is the spreading resistance stemming from the current flowing through a nanocontact, $R_{\text {sample }}$ is the sample resistance that depends on the sample (or device) geometry and measures the effect of the current path between the probe and the back contact, $R_{\text {probe }}$ is the probe resistance, $R_{\text {probe-contact }}$ is the contact resistance between probe and sample, and $R_{\text {back- }}$ contact is the back contact resistance. Particularly for silicon, the application of a high force during the measurement suppresses the contribution of $R_{\text {probe-contact }}$ by many orders of magnitude. This dramatic decrease in the contact resistance originates from the formation of the $\beta$-Sn (metallic) $S i$ pocket underneath the probe ${ }^{23,24}$. Please note that normally the contribution of $R_{\text {probe-contact }}$ to the total measured resistance is negligible in a typical SSRM experiment. However, in our case the confinement and sample heterogeneity ( $\mathrm{Si}$ on $\left.\mathrm{SiO}_{2}\right)$ may impact the $\beta$-Sn formation. Thus, the assumption of negligible $R_{\text {probe-contact }}$ may no longer be justified. Now that we have listed the different parasitic resistances present in our system, we can attempt to understand their individual contribution to the total resistance as measured in figure $2 \mathrm{~b}$. Since the probe $\left(R_{\text {probe }}\right)$ and back contact $\left(R_{\text {back-contact }}\right)$ resistances do not change during the scalpel process, eq. (2) suggests that the possible contributors for the observed increase in the resistance can be $R_{\text {sample, }} R_{\text {spr }}$ and (or) $R_{\text {probe-contact. }}$ However, the relative contributions of each of them are still unknown. To shed some light on their individual contributions, we simulated the SSRM set up as shown in figure 1c. Based on the work of Schulze et al. ${ }^{25}$ and on the measurement of $R_{\text {probe }}$ on a platinum sample, we can obtain an average probe radius of $3.7 \mathrm{~nm}$. Therefore, the probe contact radius in all the following simulations is taken as $4 \mathrm{~nm}$.

Firstly, the behaviour of the spreading resistance is investigated. For this purpose, both the contacts (i.e. probe contact and back contact) are modelled as perfectly ohmic and the presence of the underlying oxide layer is ignored. The active dopant concentration in the $\mathrm{Si}$ layer is taken same as in the SOI sample used above i.e. $5.0 \times 10^{15} \mathrm{~cm}^{-3}$. While this is an extremely simple representation of the actual SSRM circuit, it helps us to isolate the behaviour of the spreading resistance from any other effects originating from the $\mathrm{Si} / \mathrm{SiO}_{2}$ interface and non-ideal ohmic contacts. Now, we can rewrite the eq. (2) for the simulated geometry as:

$$
R_{\text {Simulation }}=R_{\text {spr }}+R_{\text {sample }}
$$


Note here that we omitted the terms $R_{\text {probe }}$ because we did not simulate the probe geometry. This remains a constant anyway and as discussed earlier, we do not analyse the measurements that show significant tip degradation during scalpel process. Since the current will flow radially from the circular probe contact towards the outer surface of the cylinder (see figure $1 \mathrm{c}$ ), the $R_{\text {sample }}$ can thus be defined as the resistance of the region where current lines become parallel to the top surface of cylinder. With this definition of $R_{\text {sample }}$, we ignore the region where the current lines are spreading as this is incorporated in $R_{\text {spr. }}$. A schematic of the cross-section of the cylinder is shown in the inset of figure $3 a$. Therefore, the maximum value of $R_{\text {sample }}$ will be the resistance of a hollow cylinder with inner and outer radii as "a" and "R" respectively. In that case $R_{\text {sample }}$ can analytically be calculated as:

$$
R_{\text {sample }}=\frac{\rho_{\text {sample }}}{2 \pi H} \ln \frac{R}{a}
$$

Using eq. (3) and (4), we calculate $R_{\text {spr }}$ as:

$$
R_{\text {spr }}=R_{\text {simulation }}-R_{\text {sample }}=R_{\text {simulation }}-\frac{\rho_{\text {sample }}}{2 \pi H} \ln \frac{R}{a}
$$

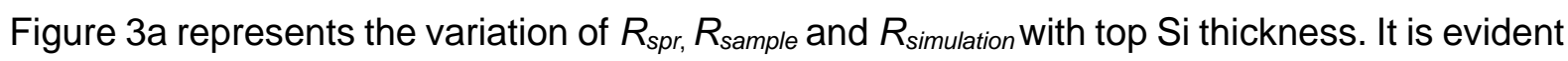
here that although the total resistance of the simulated structure increases with decreasing film thickness, the spreading resistance rather shows a continuous decrease. This is not surprising because we know that the spreading resistance is the result of the constriction to the current flow in a point contact when the current enters a very large area. As the film thickness approaches the size of the probe contact, the probe contact is no longer seen as a constriction to the current flow. In this case, current spreading occurs only at the immediate vicinity of the probe contact. While a monotonic decrease in $R_{s p r}$ is seen, the decrease becomes more significant below a critical thickness of $50 \mathrm{~nm}$. This critical thickness is not a constant value and rather depends on the probe radius. In other words, the relevant parameter would be the ratio of film thickness to the probe radius i.e. $\mathrm{H} / \mathrm{a}$. To assess the relative change of $R_{s p r}$ we normalized it with respect to the theoretical spreading resistance as defined by eq. (1) for a semi-infinite thickness of Si. This normalized $R_{s p r}\left(R_{s p r, n o r m}\right)$ is plotted against the normalized Si film thickness, $H / a$ in Figure $3 b$. Figure $3 b$ clearly shows that as the ratio $H / a$ 
approaches unity i.e. film thickness is of the size of probe radius, $R_{s p r, n o r m}$ decreases by more than $80 \%$, hence can no longer be represented by eq. (1). Note that, although for thick Si films (large $\mathrm{H} /$ a ratio) we expect $R_{\text {spr,norm }}$ to be close to unity, its maximum value was calculated to be only $60 \%$ of the theoretical value. This difference can be explained by the following two reasons. First, eq. (1) is derived for a disc shaped constriction whereas the probe contact was simulated as hemispherical. Changing the contact shape from disc to hemisphere leads to a $36 \%$ reduction in the $R_{\text {spr }}$. Second, due to the difference in placing the back contact i.e. at the curved surface of the cylinder in simulations vs at the bottom surface of the cylinder in the derivation of eq. (1), further leads to a $4 \%$ reduction in $R_{s p r}$. Nevertheless, as shown in fig 3a, the dominance of $R_{\text {spr }}$ remains intact for large $\mathrm{H} / \mathrm{a}$ and a direct correlation with $\rho_{\text {sample }}$ can be assumed. The decrease in $R_{s p r}$ with $\mathrm{Si}$ thickness is purely geometrical effect, hence samples with all doping levels will be impacted equally. Since $R_{s p r}$ decreases with film thickness, it cannot be the reason for the increase of the total resistance, hence other terms will become more important. It is indeed clear from Fig 3 a that $R_{\text {sample }}$ becomes comparable (or even greater than) to $R_{s p r}$ below $35 \mathrm{~nm}$ ( $H / a$ ratio below 9$)$ indicating that at such small dimensions the measured resistance in SSRM is no longer dominated by $R_{s p r}$ but rather by the series resistance experienced by the current flow towards the back contact. As this resistance scales with the length of the current path, the critical thickness, $H_{\text {critical }}$ at which $R_{\text {spr }}$ becomes equal to $R_{\text {sample }}$, will depend on the distance between the probe contact and the back contact which is represented by radius " $R$ " of the cylinder in our simulations. In figure 4 we show the variation of $H_{\text {critical }} / a$ as a function of back contact distance (or cylinder radius " $R$ "). This shows that by reducing the distance to back contact, the dominance of $R_{\text {spr }}$ over $R_{\text {sample }}$ can be ensured. This, however, puts very stringent requirements on the location accuracy of the back contact as $1 \mu \mathrm{m}$ from the cross section is the minimum that can be routinely achieved. From the results in figure $3 \mathrm{a}$ it is important to note that, although $R_{\text {sample }}$ increases by more than 2 orders of magnitude, the increase is definitely not enough to explain the huge increase (6 orders of magnitude) of the resistance observed in the scalpel experiment shown in figure $2 b$. 
a)

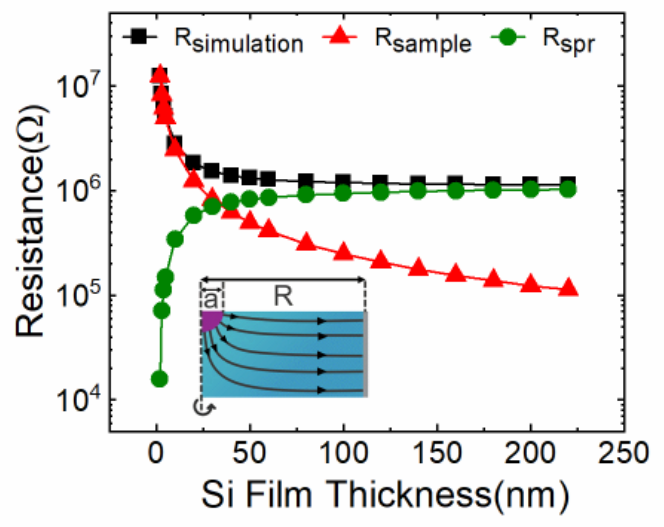

b)

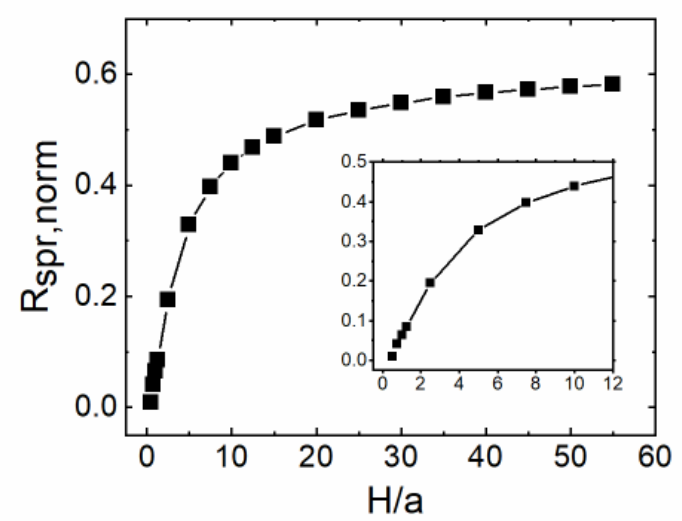

Figure3: a) Variation of total simulated resistance ( $\left.R_{\text {simulation }}\right)$, spreading resistance $\left(R_{\text {spr }}\right)$ and bulk sample resistance ( $R_{\text {sample }}$ ) as a function of top Si thickness for an ideal ohmic probe-sample contact.

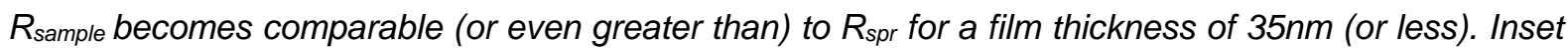
represents the cross-section of the simulated cylinder. Within a thin film, current lines become parallel to top (and bottom) cylinder surface for $r>a$, hence the resistance of the corresponding hollow cylinder represents $R_{\text {sample, }}$ which does not contribute to $R_{\text {spr. }}$ b) Variation of $R_{\text {spr,norm }}$ with decreasing film thickness (i.e. decreasing $\mathrm{H} / \mathrm{a}$ ratio) reveals that $R_{\text {spr }}$ becomes negligible when film thickness approaches to the probe contact dimensions. A zoomed-in graph is shown in the inset.

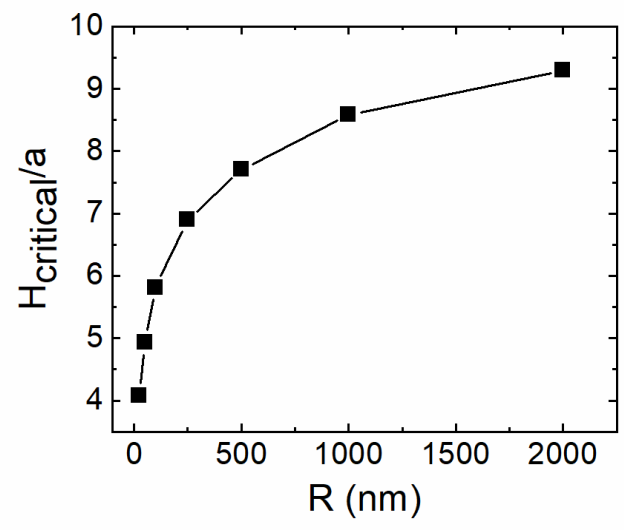

Figure 4: Variation of $H_{\text {critical }}$ a ratio as a function of cylinder radius $R$

As the results of the previous section show that $R_{\text {spr }}$ and $R_{\text {sample }}$ are not the main contributors to the huge increase in $R_{\text {Total, }}$ additional phenomena need to be explored. As discussed previously, $R_{\text {probe-contact }}$ is very low in standard SSRM as the application of a high force leads to a structural phase transformation in the underlying $\mathrm{Si}^{23}$. This phase transformation is a mechanical phenomenon, which depends on the mechanical properties and the stress distribution within the indented system. If the confinement and/or heterogeneity of the system leads to a different stress distribution, the afore mentioned phase transformation might not 
happen, thus impacting $R_{\text {probe-contact }}$ significantly. With respect to the present scalpel experiment, two effects need to be considered. Firstly, it is possible that with decreasing film thickness, the intrinsic elastic properties of Si will change. Secondly, the final stress distribution is the result of a complex interplay between the top $\mathrm{Si}$ layer and the underlying $\mathrm{SiO}_{2}$ layer that may also impact the mechanical response of the top Si layer towards the applied force ${ }^{26}$. As the B-Sn transformation leads to a dramatic change in $R_{\text {probe-contact, the measurement of }}$ resistance-force curves becomes a very sensitive way to assess the occurrence of the phase transformation. To probe the impact of film thickness on the phase transformation, we thus used resistance force curves. For this purpose, we first bevelled the SOI sample to obtain a large area with varying thicknesses and then collected resistance-force curves on the bevelled surface. The Beam-exit cross-sectional polishing $(B E X P)^{27-29}$, a variant Ar-ion milling technique, was used to obtain a smooth surface on the bevel with an angle of $8^{\circ}$. A schematic of such a cross-sectioned surface along with the measurement procedure is shown in figure 5a. As depicted, an area of $2 \times 2 \mu m^{2}$ on the bevel is divided in $128 \times 128$ pixels in $X$ and $Y$ directions. Resistance-force curves i.e. the evolution of measured resistance as a function of applied force on the cantilever, are measured on these pixels for various film thicknesses. In figure $5 b$ we show the resistance force curves obtained on all these $128 \times 128$ points, where each force curve represents the average of 128 resistance-force curves taken along the $Y$ direction. This way we obtain 128 resistance-force curves each representing a different thickness of the top Si layer. The thickness at each of these pixels can be calculated using the bevel angle and the maximum film thickness. When all these 128 curves are plotted together (see figure $5 b$ ), a similar behaviour is observed for all the film thicknesses above $11 \mathrm{~nm}$. An average of all the resistance force curves obtained for thicknesses larger than $11 \mathrm{~nm}$ is also overlaid in a solid red line. Since a sharp transition from high to low resistance at a critical force (here $\sim 1 \mu \mathrm{N}$ ) represents the drop in $R_{\text {probe-contact, we conclude that despite a very different }}$ behaviour for less than $10 \mathrm{~nm}$ thick Si films, no change can be expected in $R_{\text {probe-contact }}$ for film thicknesses greater than $11 \mathrm{~nm}$ as long as the applied force is maintained at a value greater than the critical force. Looking closely at figure $2 b$, it is found that during scalpel measurements $R_{\text {Total }}$ starts increasing for film thicknesses smaller than $80 \mathrm{~nm}$. In fact, $R_{\text {Total }}$ was measured to be already 4 orders of magnitude higher at $10 \mathrm{~nm}$ thick film, which, as inferred from figure $5 \mathrm{~b}$, cannot be attributed to $R_{\text {probe-contact }}$ 
a)

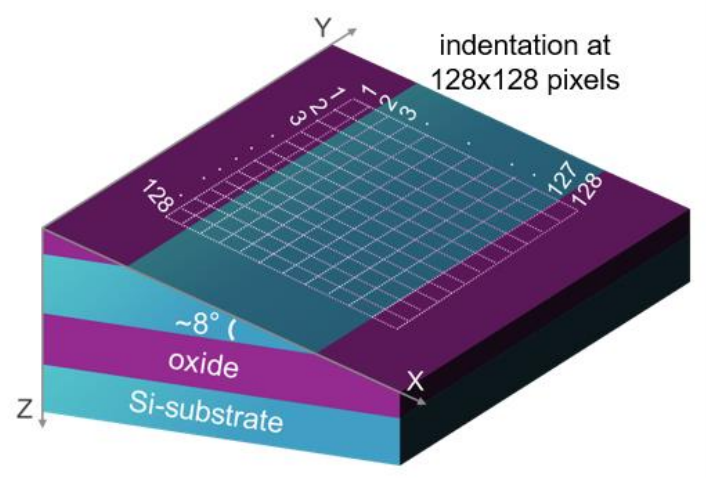

b)

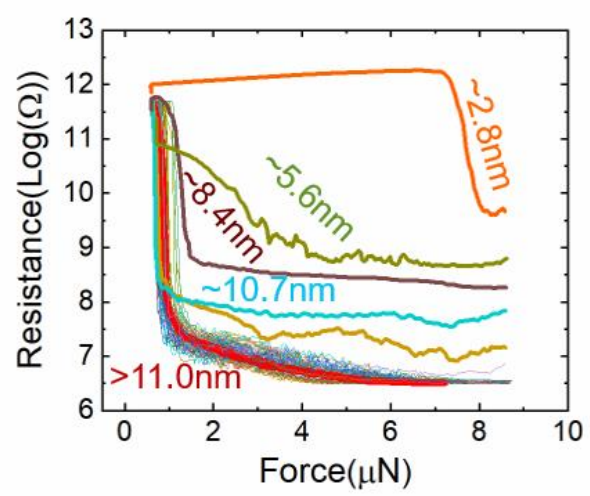

Figure 5: a) Schematic of the bevelled SOI sample. To avoid the rounding effects and protect the concerned area, an extra 400nm of oxide layer was deposited before polishing. b) Resistance force curves at various Si thicknesses are plotted. Multiple curves (thinner lines) for film thickness greater than $11 \mathrm{~nm}$ are averaged to obtain the thick solid red curve. The thickness for each curve is indicated in the corresponding color.

All the measurements and simulations done so far have indicated that none of the resistive components included in eq. (2) can lead to the observed large increase in $R_{\text {Total. }}$. However, we have not yet considered the effect of $\mathrm{Si} / \mathrm{SiO}_{2}$ interface on the $R_{\text {Total. }}$. Moreover, as previously reported $^{30}$ the contact between the probe and the sample ( $\mathrm{Si}$ in our case) in SSRM is not perfectly ohmic but rather Schottky type, which also changes the total measured resistance. Both effects are studied individually through simulations by incorporating the $\mathrm{Si} / \mathrm{SiO}_{2}$ interface and by considering the probe-sample contact as a Schottky contact with a probe contact work function taken at $0.75 \mathrm{eV}$ higher than the Si valence band. The simulation results, shown in figure $6 a$, reveal that the onset of the resistance increase is changed by the insertion of an ideal oxide interface, while the incorporation of Schottky type probe contact leads to an upward shift of the resistance. One must note that although both factors only have a limited impact on the resistance value, they do influence the carrier distribution in the film. Figure $6 \mathrm{~b}$ shows the 2D hole density map for a free standing $50 \mathrm{~nm}$ thick Si film (with ohmic probe contact) whereby a uniform distribution of holes is observed. The addition of a $\mathrm{SiO}_{2}$ layer underneath the $\mathrm{Si}$ film, as shown in figure $6 \mathrm{c}$, leads to a non-uniform distribution of carriers (holes) with a decrease below the probe and above the oxide interface. It is commonly known that when two materials with different Fermi levels are placed in contact with each other, charge is transferred until their Fermi levels align. The total amount of transferred charge is a constant value determined by the work function difference (from $0.32 \mathrm{eV}-0.42 \mathrm{eV}$ for $\mathrm{p}$-Si) between the two materials ( $\mathrm{Si}$ and oxide in this case). This implies that for a thick Si layer the depletion thickness is negligible 
and its effect on the overall current is minimal as it can easily flow far from the depleted layer. In this case $R_{\text {simulation }}$ does not show any significant dependence on the presence of the $\mathrm{Si} / \mathrm{SiO}_{2}$ interface above the critical thickness of $20 \mathrm{~nm}$ (see the zoomed in graph in the inset of figure 6a). However, when reducing the thickness of the Si film on top of the oxide, the transferred charge is redistributing. Therefore, in thin layers $(<20 \mathrm{~nm})$ the absence of the bulk material forces the current to flow through the depletion layer, resulting in a higher overall resistance of the system. This is illustrated in figure $7 \mathrm{a}$ where we show the hole density maps as simulated for $20 \mathrm{~nm}, 10 \mathrm{~nm}$ and $5 \mathrm{~nm}$ Si films when oxide is present underneath them. The $5 \mathrm{~nm}$ film is significantly depleted as compared to the $20 \mathrm{~nm}$ film effectively reducing the conductive film thickness and giving rise to a $50 \%$ increase in $R_{\text {simulation. }}$ The distribution of hole density along the dotted black and white lines (in figure7a) for various film thicknesses is also presented in figure $7 \mathrm{~b}$ and $7 \mathrm{c}$. It is evident from this graph that due to the charge transfer phenomenon occurring at the $\mathrm{Si} / \mathrm{SiO}_{2}$ interface a thin film will be far more depleted than a thicker film.

a)

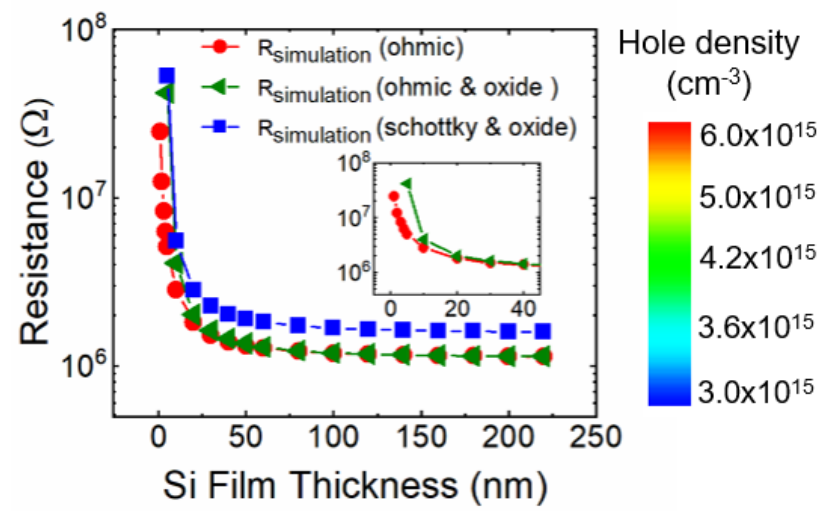

b)

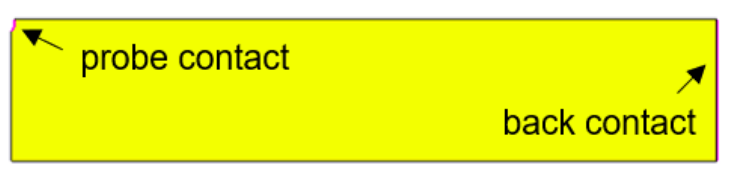

c)

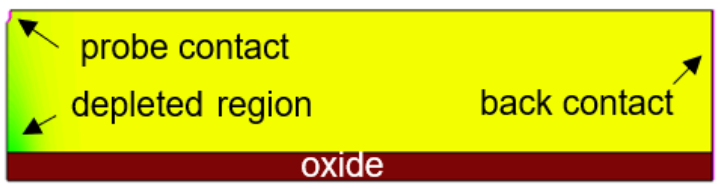

Figure 6: a) Rsimulation as a function of Si film thickness. With the introduction of oxide layer underneath, an early onset of resistance increase is observed (green triangles). Overall $R_{\text {simulation }}$ is increased in the presence of oxide (as compared to the resistance of a free-standing Si film shown in red circles. Addition of a Schottky type contact into this system (blue squares) led to an overall upward shift of $R_{\text {simulation. }}$ b) Hole density maps for 50nm thick Si film with (b) and (c) without oxide layer (shown in maroon). Regions near probe contact are slightly depleted when the oxide interface is introduced. These simulations are performed at a bias of $0.5 \mathrm{~V}$ (same as in experiments). 
a)

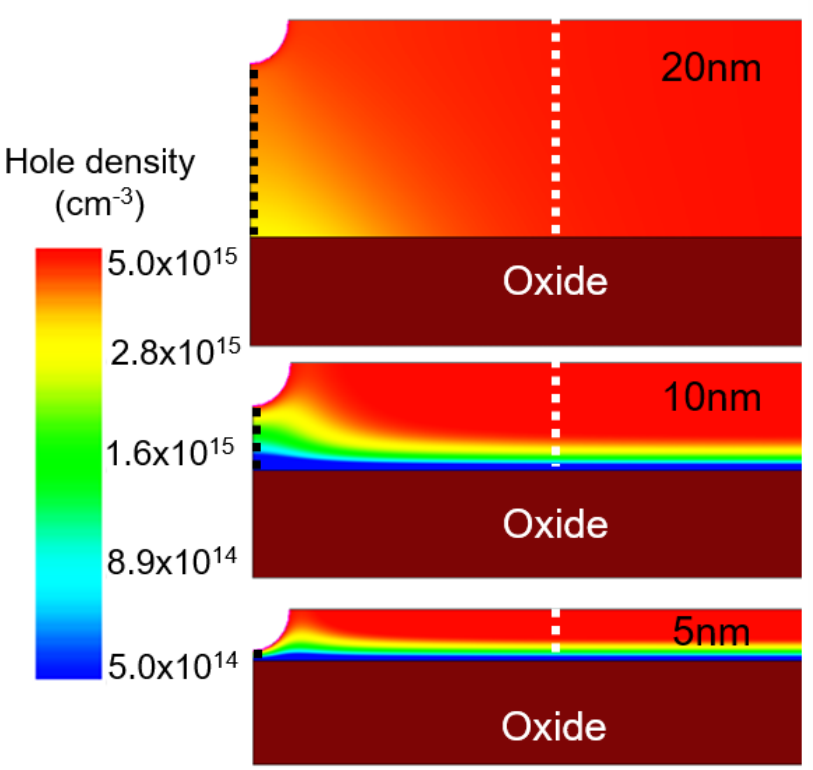

b)

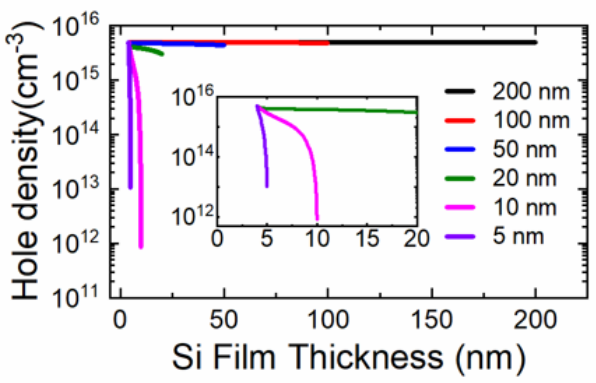

c)

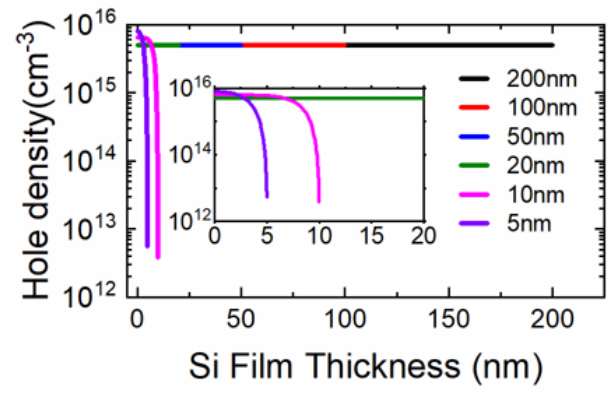

Figure 7: a) Hole density maps for 20nm, $10 \mathrm{~nm}$ and $5 \mathrm{~nm}$ Si films shows the distribution of depleted regions near the $\mathrm{Si} \mathrm{SiO}_{2}$ interface. The line profiles for hole density along b) black and c) white dotted lines show that reducing Si film thickness causes the redistribution of carriers in a way that the extent of depletion increases.

So far, we have only considered the ideal $\mathrm{Si} / \mathrm{SiO}_{2}$ interface. A point which still needs to be considered is the effect of interface states with a characteristic concentration $\left(D_{i t}\right)$ and energy distribution. We added to our simulations the concentrations $\left(D_{\text {it }}\right)$ of donor and acceptor type traps as $3 \times 10^{11} \mathrm{eV}^{-1} \mathrm{~cm}^{-2}$ and $5 \times 10^{12} \mathrm{eV}^{-1} \mathrm{~cm}^{-2}$, respectively. The $D_{\text {it }}$ distribution within the bandgap was taken as Gaussian type with donor peak at $E_{v}+0.16 \mathrm{eV}$ and acceptor peak at $E_{c^{-}}$ $0.1 \mathrm{eV}$ with sigma values equal to $0.1 \mathrm{eV}$. These values are in line with the commonly reported values for interface traps between silicon and thermally grown silicon dioxide ${ }^{31-33}$. The dependence of $R_{\text {simulation }}$ (total resistance of the simulated system with Schottky type probe contact) on film thickness is now compared with the experimental data in figure 8 . We see that upon introducing these traps, the simulation shows good agreement with the experimental data. Figures $8 \mathrm{~b}, 8 \mathrm{c}$ and $8 \mathrm{~d}$ represent the hole density maps in the presence of underlying oxide layer for $5 \mathrm{~nm}, 10 \mathrm{~nm}$ and $20 \mathrm{~nm}$ thick films, respectively. As the film thickness is reduced, the extent of the depletion region is increased. In fact, the entire film is depleted i.e. the carrier concentration is reduced by more than $\sim 2$ orders of magnitude, in the $5 \mathrm{~nm}$ case. 
b)

a)

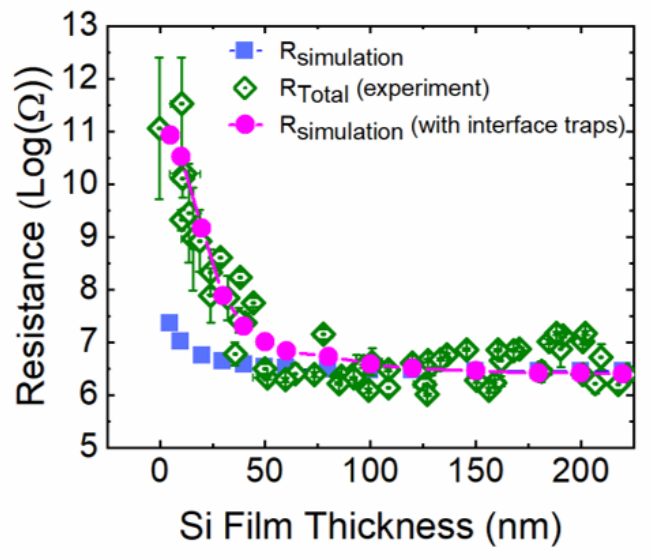

Hole density $\left(\mathrm{cm}^{-3}\right)$

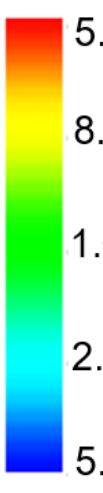

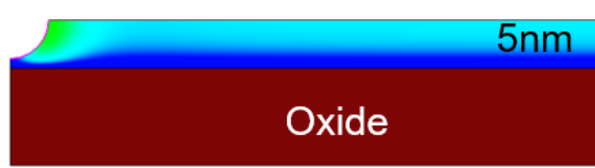

c)

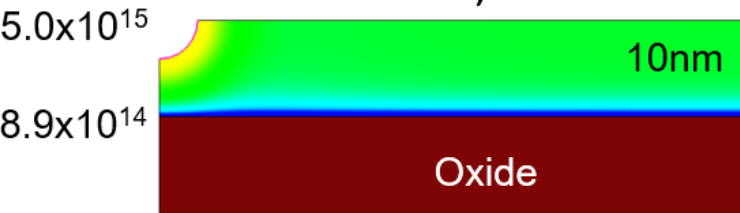

d)

$1.6 \times 10^{14}$ $2.8 \times 10^{13}$ $20 \mathrm{~nm}$ $5.0 \times 10^{12}$

Figure 8: a) The total simulated resistance, $R_{\text {simulation }}$ for various Si film thicknesses is compared with experimentally obtained results ( $R_{\text {Total }}$ (in green diamonds). The simulated model is in good agreement with the experiments when interface traps (pink circles) are introduced. A hole density map within a b) $5 \mathrm{~nm}$, c) $10 \mathrm{~nm} \&$ d) $20 \mathrm{~nm}$ Si film shows that as the film thickness is reduced, the layers get severely depleted, which eventually give rise to a very high $R_{\text {simulation. }}$

a)

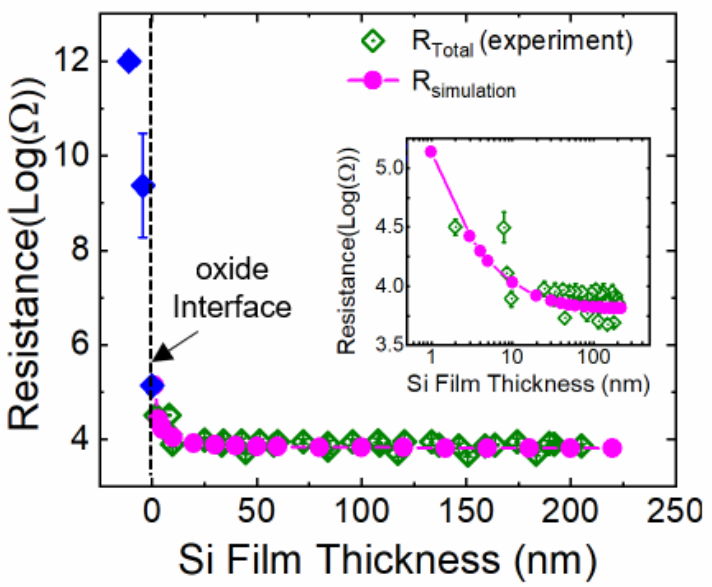

b)

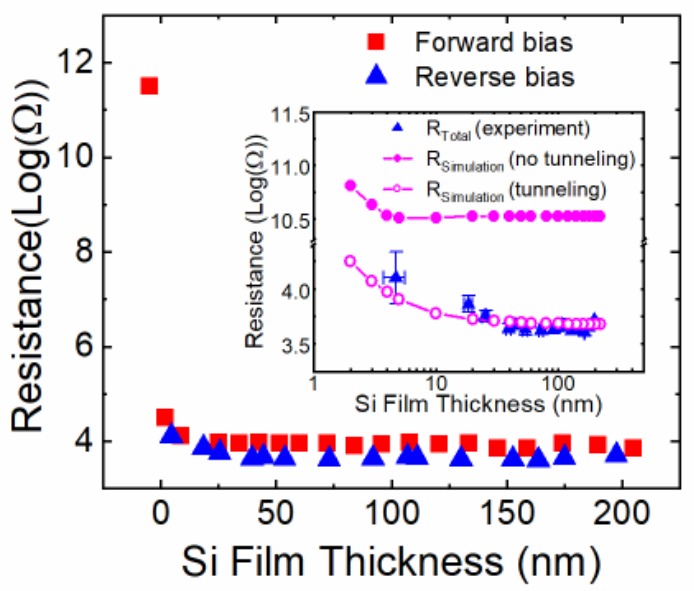

Figure 9: a)The variation of total resistance is studied on another SOI sample with relatively higher doping level i.e. $5 \times 10^{19} \mathrm{~cm}^{-3}$, both experimentally (RTotal in green diamonds) and through simulation

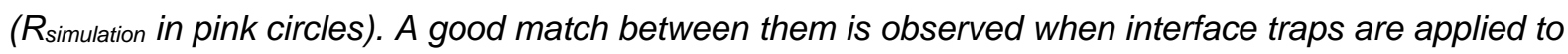
the model. A negative thickness (shown in blue diamonds) represents a scan within the oxide layer. $A$ zoom in graph is also presented in the inset. b) Scalpel experiments are performed on high doped sample under different bias conditions i.e. forward (red squares) and reverse bias (blue triangles). The similar behaviour of high doped Si under the two different polarities is explained by tunnelling current, 
which is dominant in reverse bias condition. The inset shows that the simulations show a good agreement with the experimental data when the tunnelling model is incorporated.

The density of these traps affects the onset of resistance increase and an early onset of resistance increase is observed (not shown here) if higher traps are present. Furthermore, we verified this effect by performing a similar scalpel SSRM experiments on another, similarly grown, SOI sample with relatively higher doping level (n-type Si with doping $\sim 5 \times 10^{19} \mathrm{~cm}^{-3}$ ). As expected, (see figure 9a) the effect is now reduced i.e. the measured resistance remained constant down to a thickness of $10 \mathrm{~nm}$. Also, simulations were run using the same trap density as specified previously and again a very good agreement is found. Since these trap densities are prone to change with growth parameters of the oxide interface, a different depth profile is expected in case of a differently processed sample.

Note that in figure $8 \mathrm{a}, \mathrm{R}_{\text {Total }}$ shows a small increase from $220 \mathrm{~nm}$ to $200 \mathrm{~nm}$. This increase is related to the surface states effect, where the high concentration of surface states provides an additional surface current, predominantly affecting the measurements on low doped $\mathrm{p}-\mathrm{Si}^{34}$. As the difference in the surface states is maximum for the first two scans (pristine versus scanned), this increase is mostly seen in the second scan. For the subsequent scans, the concentration of surface states reaches to a steady state due to which the effect is no longer seen.

The high doped sample used in this study is n-type, and since the work function of the probe contact (or work function of the metallic $B-S n$ pocket) is $0.75 \mathrm{eV}$ higher than the valence band, the effective Schottky barrier height for $n$-type Si becomes $0.35 \mathrm{eV}$. Furthermore, it has been noticed that despite the tip-sample contact shows rectifying behaviour for low doped $\mathrm{Si}$ samples, the high doped $\mathrm{Si}$ (both $\mathrm{p}$ - and $\mathrm{n}$-type) shows an ohmic like behaviour. Figure $9 \mathrm{~b}$ shows the comparison between the scalpel data obtained for high doped $\left(5.0 \times 10^{19} \mathrm{~cm}^{-3}\right) \mathrm{n}-\mathrm{Si}$ in forward ( $-0.5 \mathrm{~V}$ applied to the sample) and reverse bias conditions ( $0.5 \mathrm{~V}$ applied to the sample). It is clearly seen that high doped $n$-Si shows near identical behaviour for both forward and reverse bias condition. Although it is not indicated here, high doped p-Si also exhibit similar behaviour as explained in another study ${ }^{35}$. This near ohmic behaviour of tip-sample contact in high doped $\mathrm{Si}$ is explained in figure 10 with the help of energy band diagrams. In forward bias condition (see figure $10 \mathrm{a}$ ), we see that the Schottky barrier disappears (at $0.5 \mathrm{~V}$ bias), and thus electrons can easily flow from the conduction band $\left(E_{C}\right)$ to the $B-S n$. For smaller sample bias, the barrier height is lowered, and thermionic emission of electrons over the reduced barrier occurs from the conduction band to the ß-Sn. However, the higher conductivity in reverse bias can be attributed to the quantum mechanical tunnelling of electrons. In figure $10 \mathrm{~b}$, we see that due to the sharp band bending the free electrons can tunnel through the 
narrow barrier from B-Sn to the conduction band. As the band banding is gradual in low doped $\mathrm{Si}$, such tunnelling current is not efficient, thus higher resistance is observed. All scalpel experiments (both $\mathrm{p}$-Si and $\mathrm{n}$-Si) presented in figure 8a and 9a were performed under forward bias condition, thus no additional tunnelling model was required in the simulation. However, one must extend the model to account for tunnelling current while studying high doped samples under reverse bias condition. An example is given in figure 9b, where we show that the simulated model is in quantitative agreement with the experimental data in reverse bias case only when the tunnelling through the Schottky barrier is implemented. Note that the SSRM measurements in high doped regions are significantly affected by the tip resistance. Therefore, we added the average tip resistance $(3.55 \log (\Omega))$, as measured on a metal surface, to $\mathrm{R}_{\text {simulation }}$ before comparing it directly with the experimental data.

a)

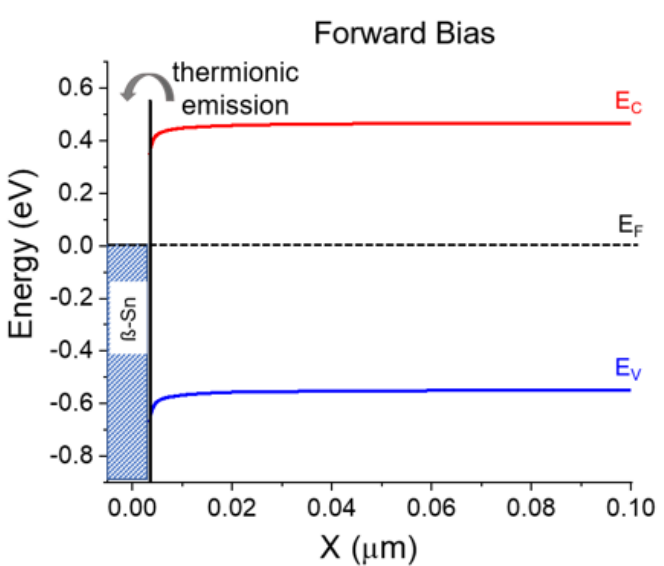

b)

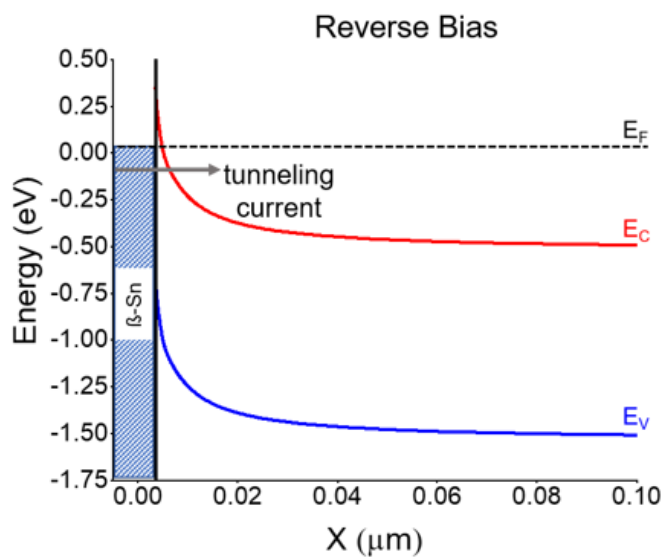

Figure 10: The energy band diagrams for $n$-Si at a) forward (-0.5 V is applied to the sample) and b) reverse bias conditions ( $0.5 \mathrm{~V}$ is applied to the sample). These band diagrams are obtained when work function of metallic B-Sn pocket is taken as $4.42 \mathrm{eV}$.

Note that, since the Si surface is continuously scanned under high stress conditions, leading to a significant surface damage ${ }^{34}$, a question can be asked if this surface damage also causes the increase in the measured resistance. For this, we must understand that the SSRM measurements are taken under high load conditions at which Si undergoes a metallic phase transformation and provides a low resistance path to the current flow. The damaged layer present on the top is simultaneously removed during scanning, thus its impact on the measured resistance is unlikely. Moreover, the scalpel SSRM performed on a uniformly doped blanket Si wafer (without oxide interface) shows no such increase in the resistance ${ }^{36}$, which further confirms that the introduced damage does not increase the resistance measured during the scalpel process. 
From the above results we can state that the quality of Si interface will play an important role in determining the SSRM results in a confined geometry. We have shown that as the material is eroded away, the extent of the depletion increases and eventually leads to a fully depleted layer. Therefore, it can be concluded that interface traps alter the effective carrier concentration measurement in a confined geometry. On a side note, this is only true if the scalpel process disrupts the depleted region near the interface, i.e. scalpeling towards the interface. When, for instance, measuring perpendicular to the $\mathrm{Si} / \mathrm{SiO}_{2}$ interface, one will still measure the impact of the interface traps, but it would reflect the actual carrier density as it is not impacted by the scalpeling measurement itself. Back to our results, the interface effect has a major impact mainly on low doped regions because of a thicker depletion region. Moreover, in the absense of traps, $R_{\text {sample }}$ plays a significant role and dominates over $R_{s p r}$, typically for $H / a$ ratio smaller than 9. For $\mathrm{H} / \mathrm{a}$ ratio larger than 9 , the increase in $\mathrm{R}_{\text {sample }}$ leads to an overall increase in the $R_{\text {Total }}$ by less than $25 \%$, which is a nominal error in SSRM measurements, meaning that below this critical ratio, the carrier quantification will not be realiable if calibrated against a bulk sample. Although for a fixed probe contact radius this critical $\mathrm{H}_{\text {critical }}$ a ratio (see figure 4) can be decreased (i.e. the critical thickness below which the $R_{\text {sample }}$ dominates over $R_{s p r}$ is reduced) by placing the back contact closer to the probe contact. The practical limit for this distance is defined by the limitations of the FIB process, which is typically used to make back contacts as close as possible to the device under test. As this effect is purely geometrical, this limit in the film thickness will affect the measurements on all doping levels equally. Below $10 \mathrm{~nm}$, an additional increase in resistance would also be observed originating from the probecontact. It is speculated that the mechanical response of the thin films towards high pressure changes (as amorphous $\mathrm{SiO}_{2}$ is a softer material compared to crystalline $\mathrm{Si}$ ), leading to more of a gradual decrease (as a function of force) in resistance instead of a very sharp decrease usually observed in thicker $(>10 \mathrm{~nm})$ films when inducing the phase transformation. The quantification of carriers in films thinner than $10 \mathrm{~nm}$ would require a calibration curve obtained at the same thickness and same material present underneath. As the mechanical response of a heterogeneous system depends on the elastic properties of the materials present and also their configuration during the resistance measurement i.e. soft film on hard substrate or hard film on soft substrate ${ }^{26}$, the limitation arising from the increase in $R_{\text {probe-contact }}$ (below $10 \mathrm{~nm}$ in figure $5 b$ ) might vary for a different system. Since $\mathrm{SiO}_{2}$ is softer than $\mathrm{Si}$, we expect that a better tolerance is achievable i.e. no increase in $R_{\text {probe-contact }}$ even below $10 \mathrm{~nm}$ if a material harder than $\mathrm{Si}$ is placed underneath. The extension of this technique towards 3D devices, which have multiple interfaces, becomes more complex as the electrical response of the probed region will depend on the mechanical properties of the surrounding material (or combination of different materials). 


\section{Conclusion}

In this work we have conducted a detailed study to understand the role of various resistive components that can affect SSRM measurements on 2D confined volumes. We have shown that the interface traps are the main contributor to the observed increase in resistance in thin layers. The reason for this was found to be the formation of a depletion region near the interface whose thickness varies with Si film thickness and original carrier concentration, ultimately leading to very high measured resistances obscuring the spreading resistance. The intrinsic resistance of the sample affects the SSRM measurements as well, although less significant. Furthermore, we observed that for a thin film, the dominance of $R_{\text {spr }}$ can be ensured by maintaining the ratio of film thickness to probe contact larger than 9 in case the back contact is located 1um away from the area of interest. This implies that, in order to probe a very thin layer quantitatively, the probe radius must be at least 9 times smaller than the film thickness or by placing the back contact closer. Additionally, $R_{\text {probe-contact }}$ is shown to increase significantly below $10 \mathrm{~nm}$ thickness in the $\mathrm{Si} / \mathrm{SiO}_{2}$ system. This effect is attributed to the change in the mechanical response of the system towards the $B-S n$ phase transformation and thus $R_{\text {probe- }}$ contact depends on the thickness of the $\mathrm{Si}$ layer and on the elastic properties of the underlying/surrounding material. Overall, we have shown that many different phenomena play when performing SSRM measurements on confined volumes and hence, we need to be careful in interpreting the SSRM data originating from state-of-the-art devices.

\section{Acknowledgements}

Authors acknowledge IMEC's Industrial Affiliation Program for funding this work. AJR thanks Oleg Kolosov and Manus Hayne for scientific discussion and use of equipment.

\section{Data availability}

The data that support the findings of this study are available from the corresponding author upon reasonable request.

\section{References}

1 J.K. Prüßing, G. Hamdana, D. Bougeard, E. Peiner, and H. Bracht, J. Appl. Phys. 125, 085105 (2019).

${ }^{2}$ L. Zhang, H. Tanimoto, K. Adachi, and A. Nishiyama, IEEE Electron Device Lett. 29, 799 (2008).

${ }^{3}$ L. Zhang, K. Ohuchi, K. Adachi, K. Ishimaru, M. Takayanagi, and A. Nishiyama, Appl. Phys. Lett. 90, 192103 (2007).

${ }^{4}$ L. Wang, V. Sallet, C. Sartel, and G. Brémond, Appl. Phys. Lett. 109, 092101 (2016).

${ }^{5}$ R. Elpelt, B. Zippelius, S. Doering, and U. Winkler, Mater. Sci. Forum 897, 295 (2017).

${ }^{6}$ S. Doering, R. Rudolf, M. Pinkert, H. Roetz, C. Wagner, S. Eckl, M. Strasser, A. Wachowiak, and T. Mikolajick, Microelectron. Reliab. 54, 2128 (2014). 
7 S. Doering, A. Wachowiak, M. Rochel, C. Nowak, M. Hoffmann, U. Winkler, M. Richter, H. Roetz, S. Eckl, and T. Mikolajick, Microelectron. Eng. 142, 40 (2015).

8 M. Lanza, Conductive Atomic Force Microscopy, 1st ed. (John Wiley \& Sons, Ltd, 2017), Chap. 8.

${ }^{9}$ U. Celano, P. Favia, C. Drijbooms, O. Dixon-Luinenburg, O. Richard, H. Bender, E. Vancoille, K. Paredis, R. Loo, and A. Schulze, in Frontiers of Characterization and Metrology for Nanoelectronics, edited by E.M. Secula and D.G. Seiler (NIST, 2017).

10 X. Ou, P.D. Kanungo, R. Kögler, P. Werner, U. Gösele, W. Skorupa, and X. Wang, Adv. Mater. 22, 4020 (2010).

${ }_{11}$ P. Eyben, M. Xu, N. Duhayon, T. Clarysse, S. Callewaert, and W. Vandervorst, J. Vac. Sci. Technol. B 20, 471 (2002).

12 P. De Wolf, T. Clarysse, W. Vandervorst, J. Snauwaert, and L. Hellemans, J. Vac. Sci. Technol. B 14, 380 (1996).

${ }^{13}$ K.D. Sattler, Fundamentals of Picoscience (CRC Press, 2013), p.677.

${ }^{14}$ W. Vanderworst and M. Meuris, U.S. Patent No. 5,369,372 (29 November 1994).

${ }^{15} \mathrm{~F}$. Llewellyn-Jones, The Physics of Electrical Contacts (Clarendon Press, 1957).

${ }^{16}$ P. Eyben, J. Machillot, M. Kim, T. Miyashita, N. Yoshida, H. Bender, O. Richard, K. Paredis, L. Wouters, J. Mitard, N. Horiguchi, R. Ritzenthaler, A. De Keersgieter, U. Celano, T. Chiarella, A. Veloso, H. Mertens, V. Pena, and G. Santoro, in Int. Electron Devices Meet. IEDM (IEEE, San Francisco, CA, USA, 2019), p. 238.

17 P. Eyben, P. Matagne, T. Chiarella, A. De Keersgieter, S. Kubicek, J. Mitard, A. Mocuta, N. Horiguchi, A.V.-Y. Thean, and D. Mocuta, in Int. Conf. Simulation Semiconductor Process. Devices, 2016, p. 287.

18 J.K. Prüßing, T. Böckendorf, G. Hamdana, E. Peiner, and H. Bracht, J. Appl. Phys. 127, 055703 (2020).

${ }^{19}$ T. Hantschel, T. Clarysse, A. Ajaykumar, F. Seidel, M. Tsigkourakos, T. Nuytten, K. Paredis, P. Eyben, B. Majeed, D.S. Tezcan, and W. Vandervorst, Microelectron. Eng. 121, 19 (2014).

20 U. Celano, F.-C. Hsia, D. Vanhaeren, K. Paredis, T.E. Nordling, J.G. Buijnsters, T. Hantschel, and W. Vandervorst, Sci. Rep. 8, 2994 (2018).

${ }^{21}$ Sentaurus Structure Editor User Guide, Synopsys, I-2016.03 ed. (2016).

22 Sentaurus Device User Guide, Synopsys, I-2016.03 ed. (2016).

${ }^{23}$ K. Mylvaganam, L.C. Zhang, P. Eyben, J. Mody, and W. Vandervorst, Nanotechnology 20, 305705 (2009).

${ }^{24}$ A. Kailer, Y.G. Gogotsi, and K.G. Nickel, J. Appl. Phys. 81, 3057 (1997).

25 A. Schulze, Ph.D. thesis, KU Leuven, 2013

${ }^{26}$ O.O. Okudur, Ph.D. thesis, KU Leuven, 2020

${ }^{27}$ A.J. Robson, I. Grishin, R.J. Young, A.M. Sanchez, O.V. Kolosov, and M. Hayne, ACS Appl. Mater. Interfaces 5, 3241 (2013).

28 O.V. Kolosov, I. Grishin, and R. Jones, Nanotechnology 22, 185702 (2011).

29 O.V. Kolosov and I. Grishin, EU Patent No. EP2537017B1 (9 November 2016).

30 P. Eyben, S. Denis, T. Clarysse, and W. Vandervorst, Mater. Sci. Eng. B 102, 132 (2003).

${ }^{31}$ H. Statz, G.A. DeMars, L. Davis Jr, and A. Adams Jr, Phys. Rev. 101, 1272 (1956).

${ }^{32}$ B.E. Deal, M. Sklar, A.S. Grove, and E.H. Snow, J. Electrochem. Soc. 114, 266 (1967).

${ }^{33}$ S. Alexandrova, A. Szekeres, and E. Halova, IOP Conf. Ser. Mater. Sci. Eng. 15, 012037 (2010).

34 R. Coq Germanicus, Ph. Leclère, Y. Guhel, B. Boudart, A.D. Touboul, P. Descamps, E. Hug, and P. Eyben, J. Appl. Phys. 117, 244306 (2015).

35 J. Mody, Ph.D. thesis, KU Leuven, (2013).

${ }^{36}$ K. Pandey, K. Paredis, T. Hantschel, C. Drijbooms, and W. Vandervorst, Sci. Rep. (to be published). 


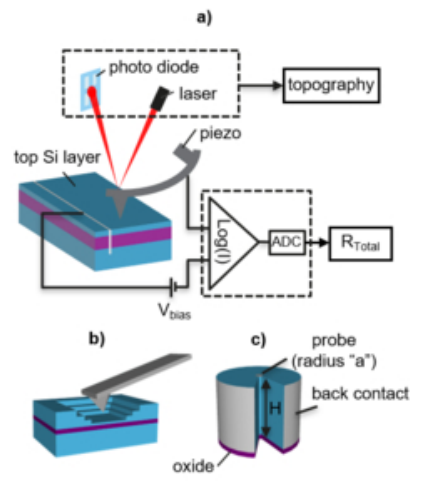



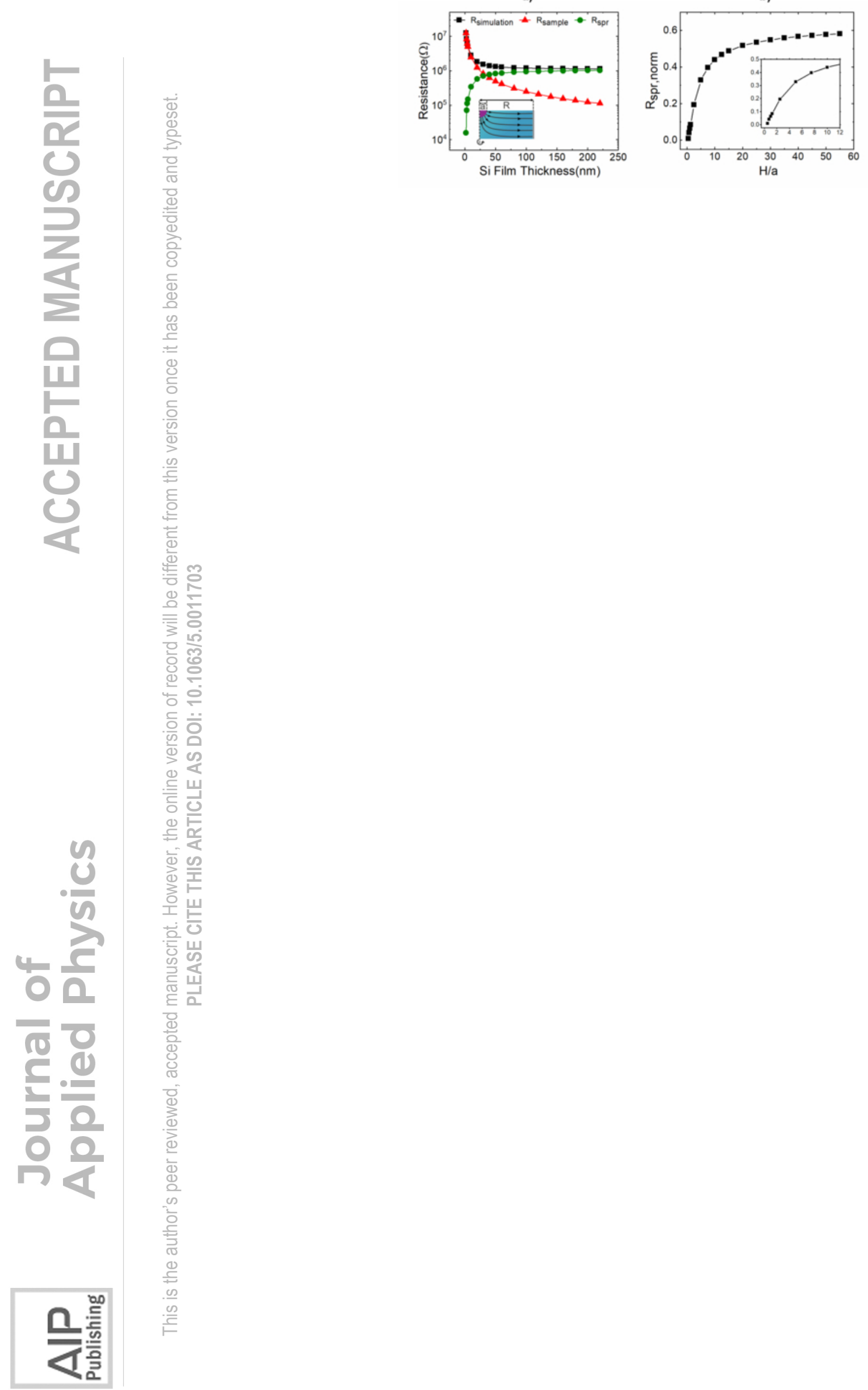

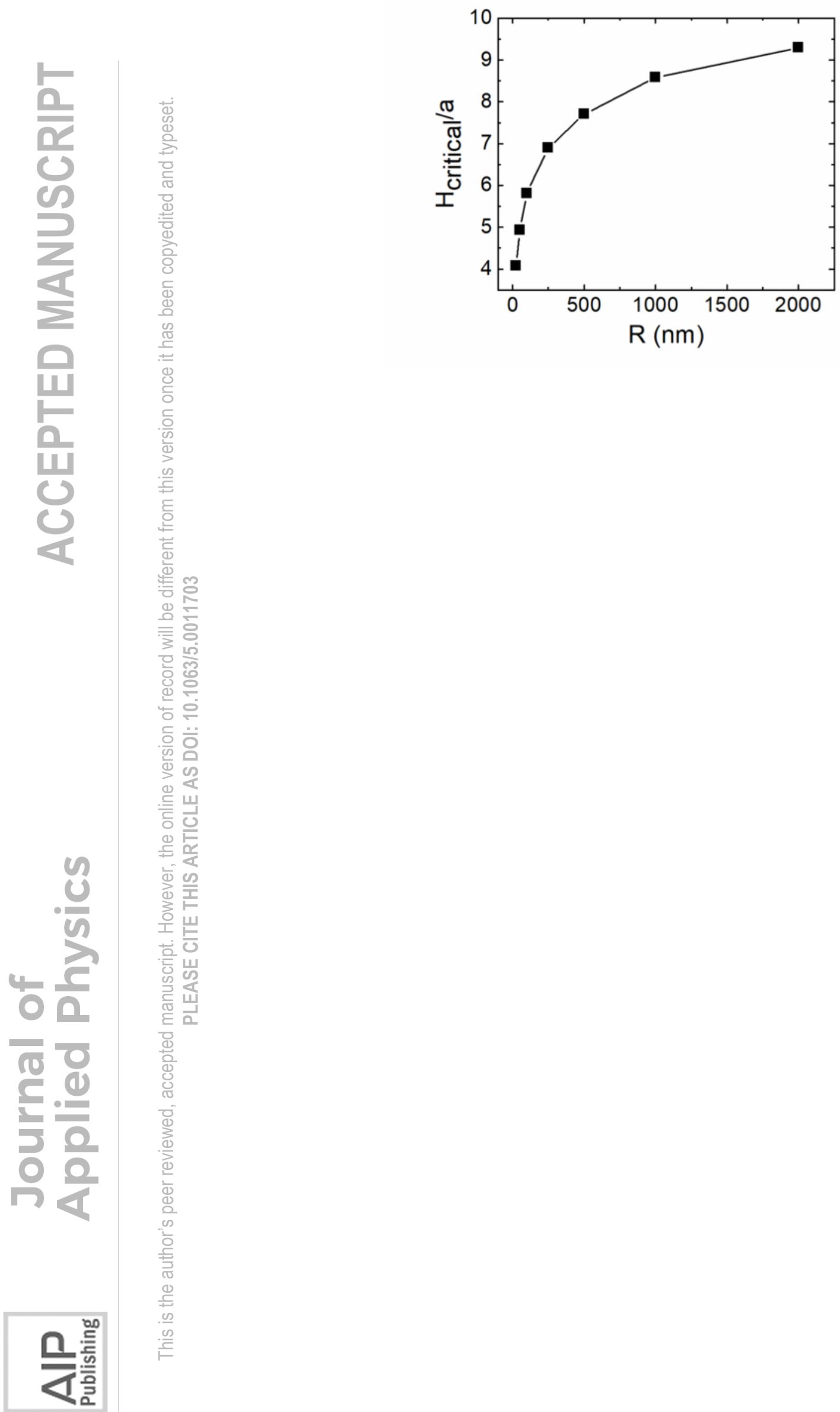

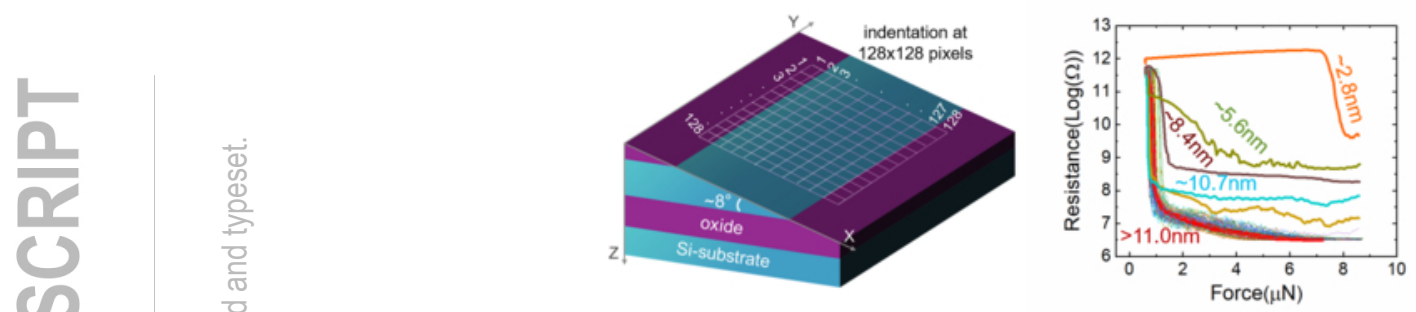

.

章

$\longrightarrow$

U1

焉

는

0

选

年

둥

눙

응

ญ인

능 은

등 흥

민

赵

ㄸ

흥

(1)

(1)

0 บ

1 오 는

(4)

( 1

(10)

(1)

닌

(อ)

응

门

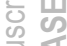

남

है

넝

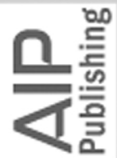



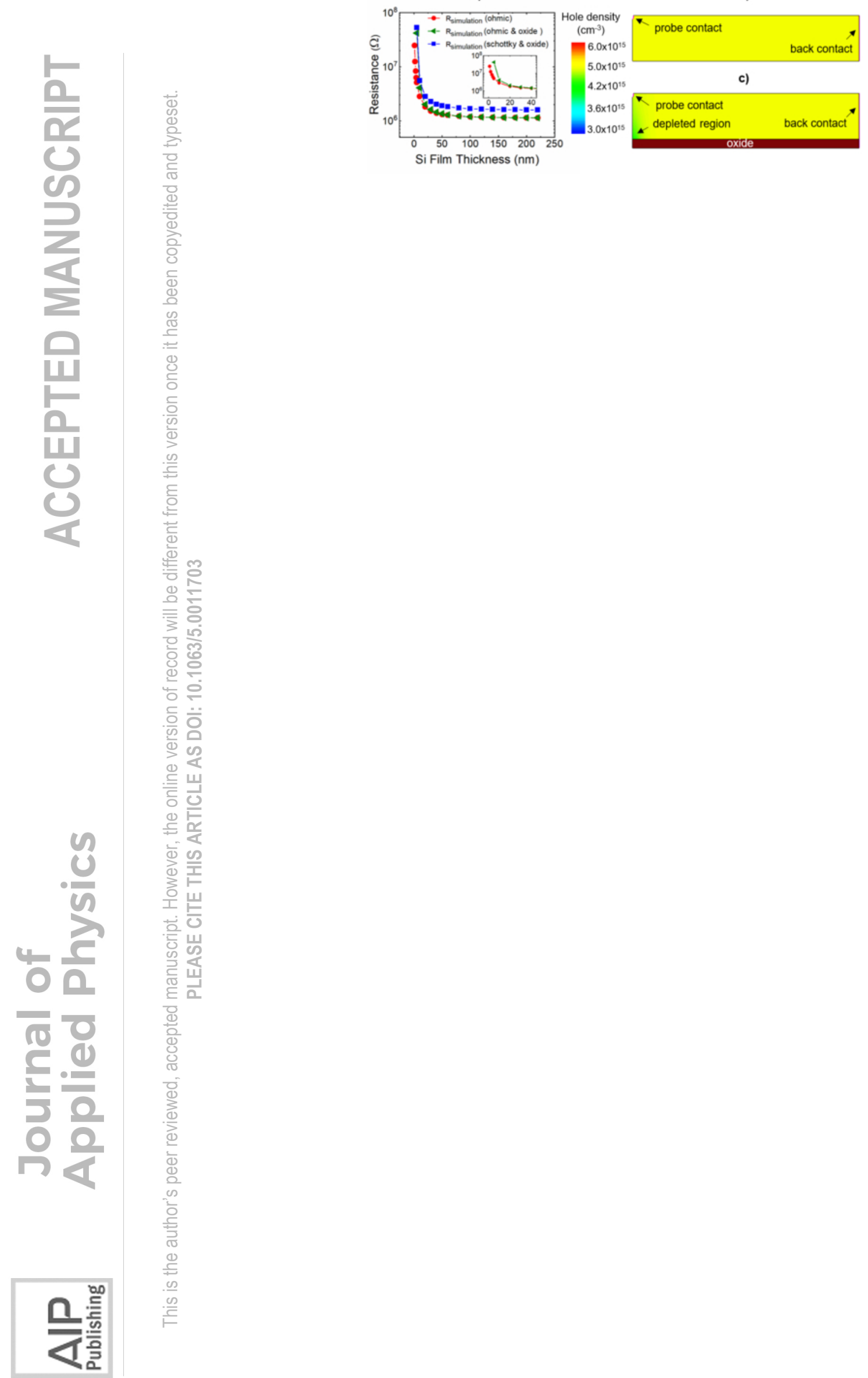
a)

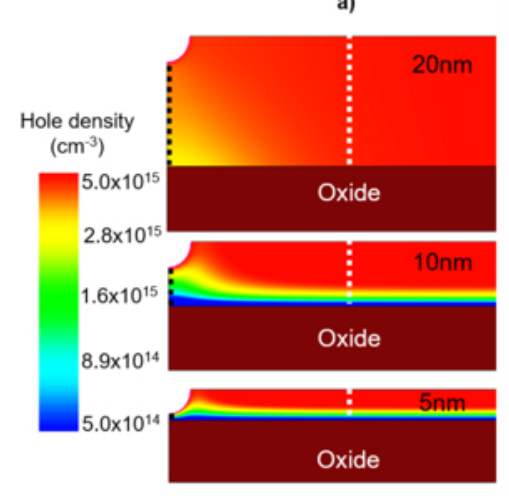

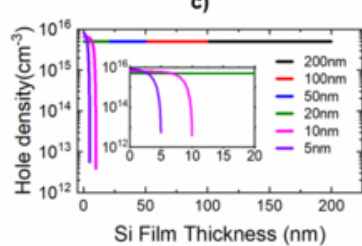


a)

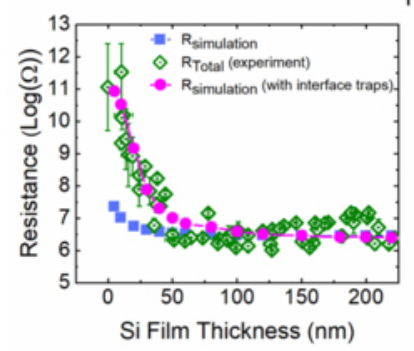

Hole density

$\left(\mathrm{cm}^{-3}\right)$

Oxide

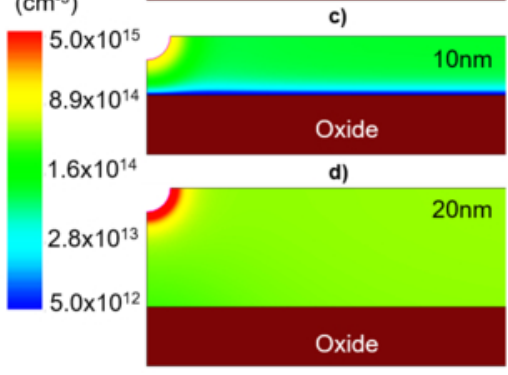

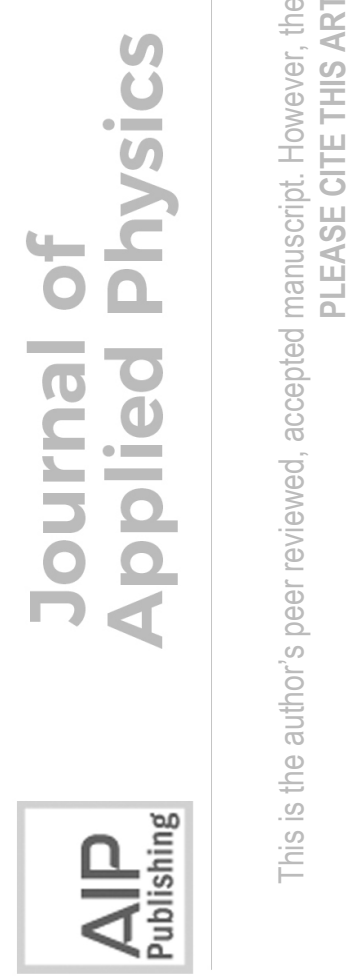



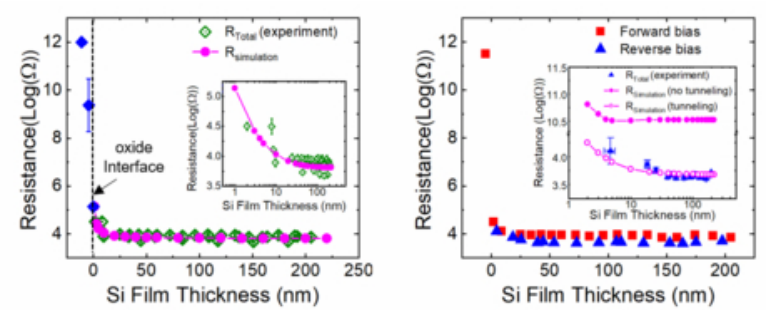

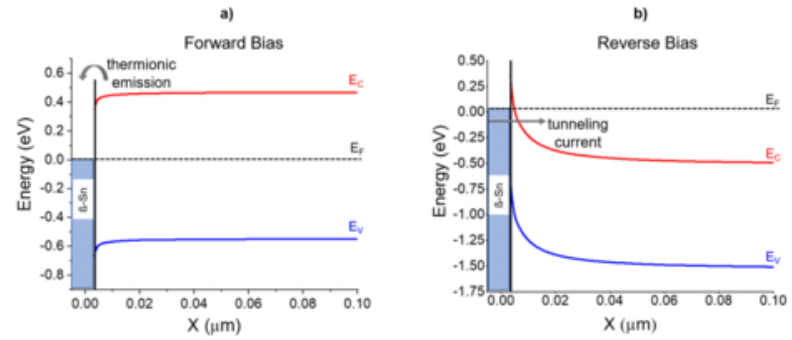

$\frac{3}{2}$

0 垃

은

흥

을둥

뇽

응 ํํㅇ

엉으

능 으

등 흠

क⿻丷ㄴ

电

뜸

ธิ

(1)

(1)

ऐ)

(

(4)

( 1.

는

ํㅜㄴ

들 荘

(10)

(1) (1)

임

(1)

(D)

( 0

1 